\title{
Status of the Mu2e experiment at Fermilab
}

\author{
Stefano Miscetti ${ }^{1}$ a on behalf of the Mu2e Collaboration \\ ${ }^{1}$ Laboratori Nazionali di Frascati dell'INFN, Via Enrico Fermi 40, 00044, Frascati, Italy
}

\begin{abstract}
The Mu2e experiment aims to improve, by four orders of magnitude, current sensitivity in the search for the charged-lepton flavor violating (cLFV) neutrino-less conversion of a negative muon into an electron. The conversion process will be identified by a distinctive signature of a mono-energetic electron with energy slightly below the muon rest mass. In the Standard Model this process has a negligible rate. However, in many Beyond the Standard Model scenarios its rate is within the reach of Mu2e sensitivity. In this paper, we explain the Mu2e design guidelines and summarize the status of the experiment.
\end{abstract}

\section{Introduction}

Since flavor violation has been established in quarks and neutrinos, it is natural to expect flavor violating processes also among the charged leptons. Albeit in the Standard Model (SM) there is not any global symmetry requiring lepton flavor violation, when massive neutrinos are introduced, the SM provides a mechanism for cLFV via neutrino-mixing in loops. In this case, the cLFV rates get suppressed by the ratio between the mass-squared difference of neutrino mass eigenstates and the $\mathrm{W}$-mass squared, thus resulting to be negligible $\left(B(\mu \rightarrow e \gamma) \leq 10^{-54}[1]\right)$. On the other hand, many theories beyond the Standard Model (BSM) predict additional particles and interactions that can enhance such processes up to a level achievable to the current generation experiments $[2,3]$.

The search for cLFV has a long history both in the muon and tau decays $(\mu \rightarrow e \gamma, \mu \rightarrow 3 e, \tau \rightarrow \mu \gamma$, $\tau \rightarrow 3 \mu$ ) as well as in the muon conversion process $(\mu N \rightarrow e N)$ in the field of a nucleus N. Muon-cLFV experiments have been undertaken since 1947 when Pontecorvo first searched for the $\mu \rightarrow e \gamma$ process. Since then, the sensitivity has improved by 11 orders of magnitude. The cLFV muon searches are of particular interest because of the possibility to carry out clean measurements free of theoretical background and the availability of high intensity muon beams. The highest sensitivity reached and the largest expected improvements still resides in the muon sector. The most stringent upper limits (reported here at $90 \%$ C.L.) have been reached at PSI:

1. $\mu \rightarrow e \gamma, \mathrm{BR}<4.2 \times 10^{-13}$ by MEG [4] in 2016 ;

2. $\mu^{+} \rightarrow e^{+} e^{-} e^{+}, \mathrm{BR}<$ by SINDRUM [5] in 1998 ;

3. muon conversion on gold, $\left(R_{\mu e}<6 \times 10^{-13}\right)$ [6] by SINDRUM-II in 2006.

\footnotetext{
a e-mail: Stefano.Miscetti@LNF.INFN.IT
}

A solid international program continues nowadays with the upgrade of MEG [7, 8], the proposed Mu3e search at PSI $[7,9]$ as well as the searches for muon to electron conversion with Mu2e at Fermilab [10] and COMET at JPARC $[7,11]$. These experiments plan to extend the current sensitivity of one or more orders of magnitude providing access to new physics mass scales in the $10^{3}-10^{4}$ $\mathrm{GeV}$ range, well beyond what can be directly probed at colliders. In case of signal observation they will indicate a clear evidence of new physics whilst in case of non observation they will pose strong limits on the development of new theories. Moreover, the complementary sensitivity of these three $\mu \rightarrow \mathrm{e}$ transition searches to new physics sources [12] offers the possibility of differentiating among various BSM models. To maximize the discovery potential, we should continue to pursue a world programme in the cLFV muon sector for current and upgraded options. This has been exploited in summer 2018 by submitting a joint cLFV report, across experiments, to the European Strategy group [13].

\section{Mu2e: $\mu$ to $e$ conversion at FNAL}

The Mu2e goal is to improve by four orders of magnitude the current best measurement on $R_{\mu e}$, the rate of neutrino-less conversion of a muon into an electron in the field of a nucleus with respect to the dominant muon capture process, so to reach a single event sensitivity of $R_{\mu e}=3 \times 10^{-17}$. The experiment technique starts by stopping a high intensity low momentum beam of negative muons in an aluminium target. The muons get trapped in orbit around the nucleus with a lifetime in the bound system $\left(\tau_{\mu}\right)$ of $864 \mathrm{~ns}$. The distinctive $\mu \rightarrow$ e conversion signature is the detection of a mono-energetic conversion electron $(\mathrm{CE})$ with momentum very close to the muon rest mass, $E_{C E}=104.96 \mathrm{MeV}$ (Fig. 1.left).

Muons stopped on aluminium have a $39 \%$ probability of undergoing a three-body decay when orbiting around 

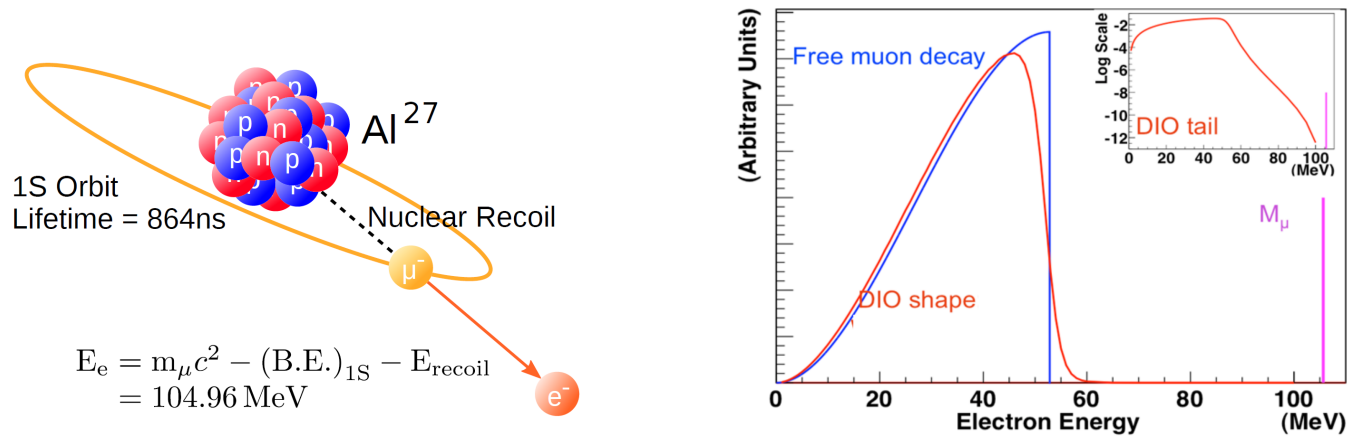

Figure 1. (Left) Sketch of the muon to electron conversion process. (Right) Energy spectrum for electrons produced from muon decays in orbit. The spectrum is a modified Michel spectrum with a long tail that falls quickly as $\left(E_{e}-E_{\text {endpoint }}\right)^{5}$ in proximity to the kinematic endpoint. Perfect resolution assumed.

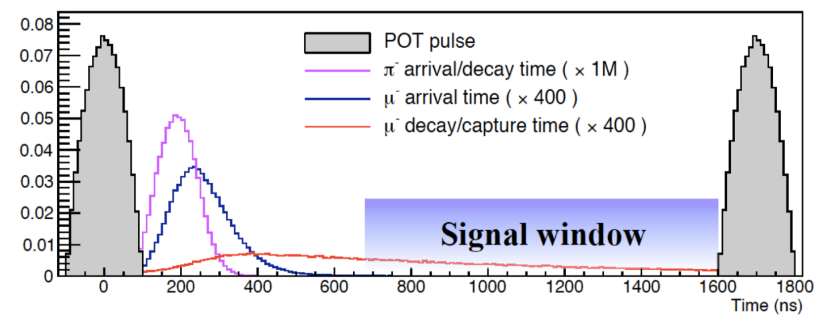

Figure 2. Sketch of the timing structure of the Mu2e beam.

the nucleus. The electron spectrum of this Decay-In-Orbit (DIO) process substantially differs from that of free decay, due to the presence of a large recoil tail that falls rapidly as the electron energy approaches the kinematical endpoint. The CE line has to be distinguished, with a high momentum resolution detector, from the DIO electron spectrum, Fig. 1.right.The remaining $61 \%$ of the stopped muons gets captured by the nucleus and becomes a source of low energy neutrons, protons and photons that creates a harsh radiation environment and increases the hit-occupancy in the detector system.

Apart from the DIO contribution, an additional background source comes from the radiative pion capture (RPC), $\pi+N \rightarrow \gamma+N^{\prime}$. Here, the electron positron pair, produced either by internal or external conversion, becomes a source of fake CE candidates when the $\mathrm{e}^{-}$momentum gets inside the signal selection window.

In order to reach the required sensitivity, the experiment has to collect $10^{18}$ stopped muons with a background below 0.5 events. These considerations have driven the design strategy of Mu2e, based on four key elements:

\section{A high intensity muon beam}

The goal is to increase the muon intensity by $10^{4}$ with respect to previous experiments and reach $10^{11}$ muons/s on target. Following an idea by Dzhilkibaev and Lobashev, proposed in 1989 for the MELC [14] experiment (later cancelled), this improvement can be achieved by means of a curved solenoidal system to allow an efficient transport, a long decay channel and charge and momentum selection. Such a selective transport channel increases muon intensity while reducing the associated beam power (compare $1 \mathrm{MW}$ for Sindrum-II and $8 \mathrm{~kW}$ for $\mathrm{Mu} 2 \mathrm{e})$.

\section{A pulsed beam structure}

The muon lifetime in the bound system for an aluminum target well matches the bunch period of the Fermilab accelerator (micro-bunch of 1694 ns period). The trick is to wait for the prompt backgrounds to decay and start the data acquisition $\sim 700$ ns after the bunch arrival time (Fig. 2).

\section{A high proton extinction requirement}

The effects of the un-synchronized particles traveling with the beam have to be negligible. The proton extinction. i.e. the ratio between the number of protons traveling in the beam in the out of time window with respect to the one in time, has to be smaller than $10^{-10}$. Calculation by full simulation has shown that this level of extinction well suppress the prompt backgrounds generated by beam electrons, in-flight-decay muons, in-flight-decay pions and RPC .

\section{A redundant high-precision detector}

This is needed to analyse the products from muon interaction on target to separate CE and DIO spectra and make the contribution from additional background sources negligible.

\subsection{The Mu2e detector layout}

The layout of the Mu2e experiment is shown in Figure 3. An array of superconducting solenoids forms a graded magnetic system composed of a Production Solenoid, PS, a Transport Solenoid, TS, and a Detector Solenoid, DS.

The PS contains a tungsten target that intercepts an 8 $\mathrm{GeV}$ pulsed proton beam brought from the FNAL delivery ring by means of a dedicated beam-line. The gradient 


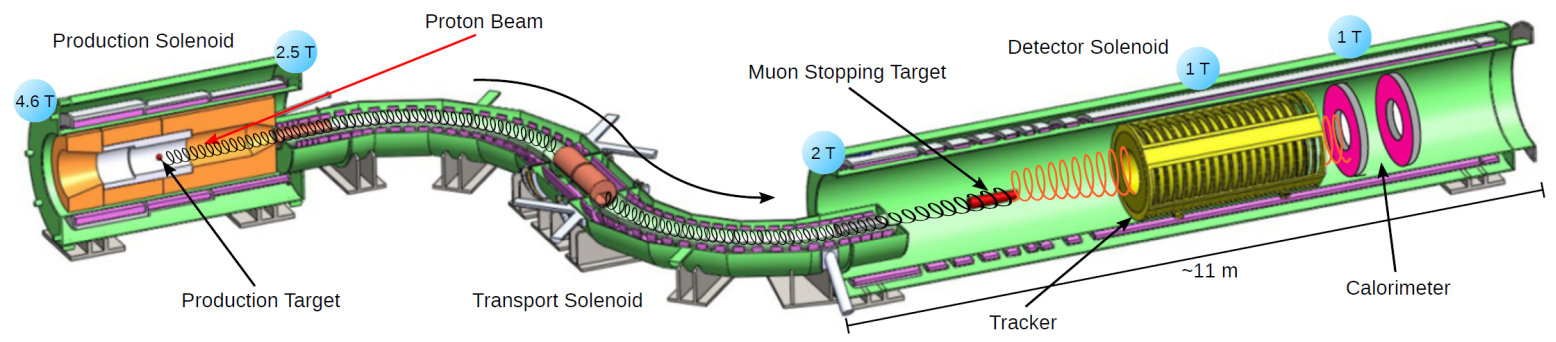

Figure 3. Layout of the Mu2e experiment.

field in the PS increases from 2.5 to 4.6 Tesla in the same direction of the incoming beam and opposite to the outgoing muon beam direction. This gradient field works as a magnetic lens focusing low energy particles into the transport channel. The focused beam is constituted by muons, pions and a small number of protons and antiprotons. After a long study, the final design of the production target is shown in Fig. 4. It is composed of $160 \mathrm{~mm}$ of segmented tungsten with four $1 \mathrm{~mm}$ thick $\times 13 \mathrm{~mm}$ tall fins ensuring support to the structure. Tungsten has been chosen as target material because of its thermal properties: the high melting point and the low thermal expansion coefficient. The new target design handles the temperature problems but reduces the achievable muon yields $(0.0018 \%$ to $0.0015 \%$ ).

The S-shaped Transport Solenoid efficiently transfers low energy, negatively charged particles to the end of beam-line while allowing for a large fraction of the pions to decay into muons. A midsection collimator attenuates nearly all positively charged particles while the antiproton residual background is reduced by a special absorber.

The DS presents a graded field from 2 to 1 Tesla in the upstream region where the muon stopping target resides. This lens increases the acceptance for $\mathrm{CE}$ while aiding in the rejection of beam-related backgrounds. A uniform axial magnetic field of 1 Tesla occupies the region of the tracker and calorimeter systems. Approximately 50\% of the muon beam is stopped by the target while the remaining beam is dumped at the end of the cryostat. The DS is covered externally by a Cosmic Ray Veto system.

The tracking detector [15] is composed of low mass straw drift tubes oriented transversally to the solenoid axis and consists of nearly 20000 straw tubes arranged in 18 stations, as shown in Fig. 5. Each tube is $5 \mathrm{~mm}$ in diameter and contains a $25 \mu \mathrm{m}$ sense wire. The straw walls are made out of Mylar and have a thickness of $15 \mu \mathrm{m}$. Straw length varies from 430 to $1120 \mathrm{~mm}$. The tracker is $\sim 3$ $\mathrm{m}$ long. It measures the momenta of the charged particles from the reconstructed trajectories using the hits detected in the straw. A central hole, $38 \mathrm{~cm}$ in diameters, makes the device blind to low momentum particles $(\mathrm{p}<55 \mathrm{MeV} / \mathrm{c})$. Groups of 96 straws are assembled into panels. In order to reduce the left-right ambiguity, each panel is composed of two layers of staggered straws. Six panels (three per side rotated by $120^{\circ}$ ) are assembled into planes. Each planes'

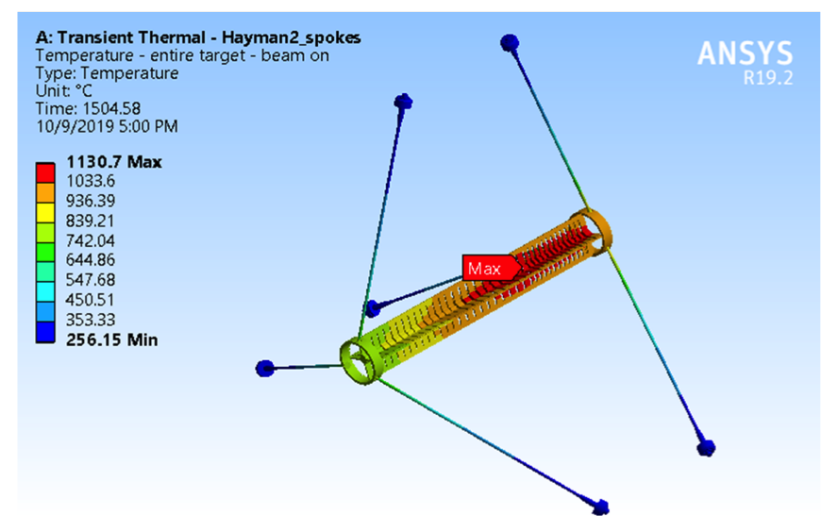

Figure 4. (Left) Final design of the Mu2e Production Target.

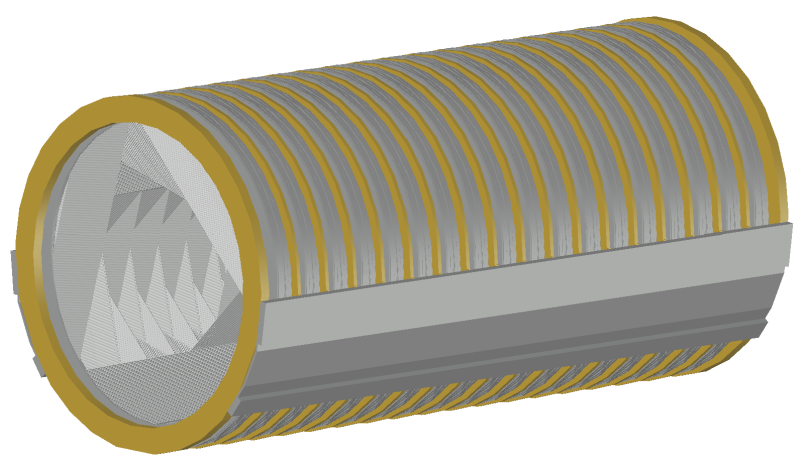

Figure 5. Sketch of Mu2e Tracker system.

face is made of three panels, rotated by $30^{\circ}$. A pair of planes makes a station; the planes are separated by $46 \mathrm{~mm}$ and are rotated of $180^{\circ}$ with respect to each other around the beam axis.

The tracking preparation is under way. The panels pre-production started in February 2019 at University of Minnesota (Minneapolis). The expected conclusion of the straw production is May 2020. The starting point of the panel production is the straw preparation: after having been laser cut, each straw undergoes a resistance, leak and length test. Then, in six days, 96 straws are assembled together to make a panel. Pictures of the different operations performed to make a panel are reported in Fig. 6 . All these procedures can be performed simultaneously on 


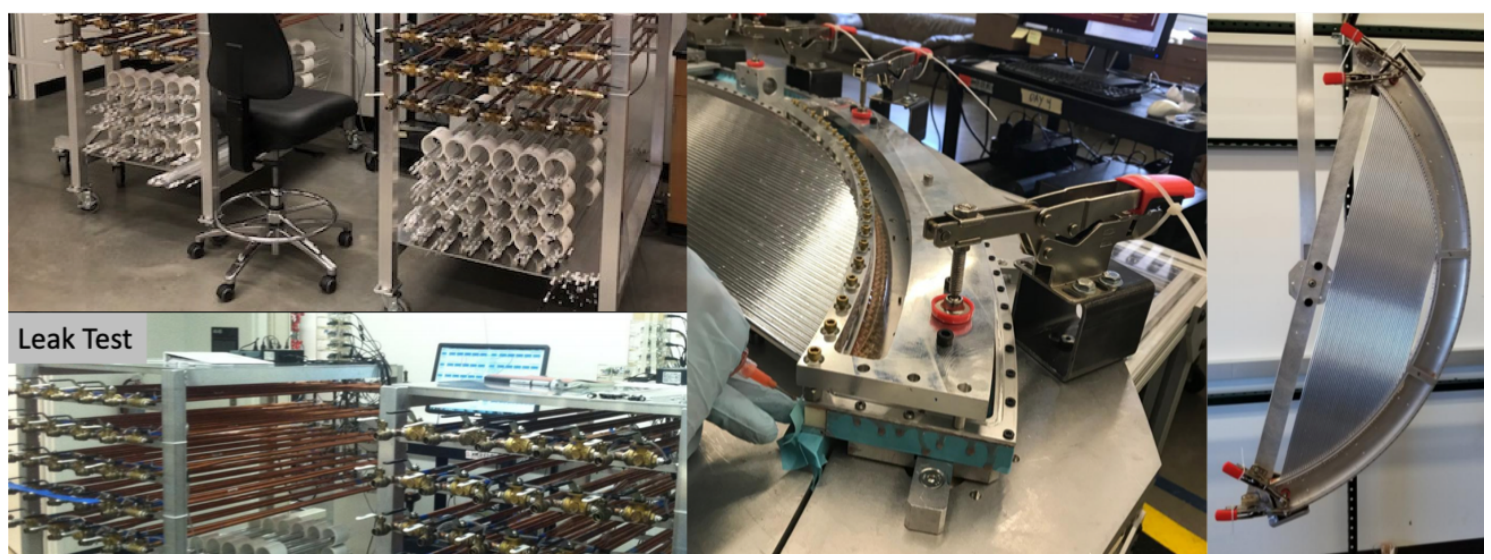

Figure 6. Left: (top) straw storage system, (bottom) leak test. Middle: panel assembly. Right: assembled panel waiting to be leak tested.

different panels so that it is possible to achieve one panel assembled per day, assuming six assembly stations working synchronously.

An eight channel tracker prototype has been built and tested with cosmics. In Fig. 7, the measured position resolution well compares with MC expectations. The data-MC shift observed is mainly due to a different T0 calibration. The overall tracker performance has been evaluated using the Mu2e full simulation. Results, shown in Fig. 8, are well within the tracking system physics requirements.

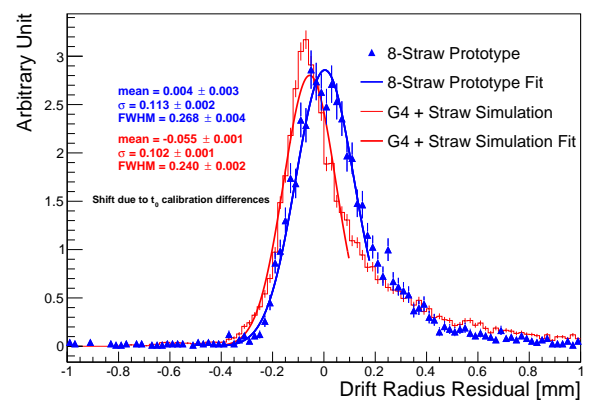

Figure 7. (Left) Comparison between data and MC position resolution for the tracker prototype as evaluated with cosmic rays.

The Mu2e calorimeter [16] has to provide: confirmation for CE signal events, a powerful $e / \mu$ separation - with a muon rejection factor of $\sim 200$, a standalone trigger and a fast seeding for track reconstruction. An energy resolution of $\mathrm{O}(10 \%)$ and a time resolution $<500$ ps for 100 $\mathrm{MeV}$ electrons are sufficient to fulfil these requirements. The calorimeter design consists of two disks (Fig. 9) made by 674 un-doped CsI scintillating crystals of $(34 \times 34 \times 200)$ $\mathrm{mm}^{3}$ dimension. Each crystal is read-out by two custom large area $\left(2 \times 3\right.$ of $6 \times 6 \mathrm{~mm}^{2}$ cells $)$ arrays of UV-extended Silicon Photo-Multipliers (SiPMs). The crystals will receive an ionizing dose of $90 \mathrm{krad}$ and a fluence of $3 \times 10^{12}$ $\mathrm{n} / \mathrm{cm}^{2}$ in three years of running. The SiPMs and the Front End (FEE) electronics, shielded by the crystals, will get a smaller dose. Equalization of the crystal response will

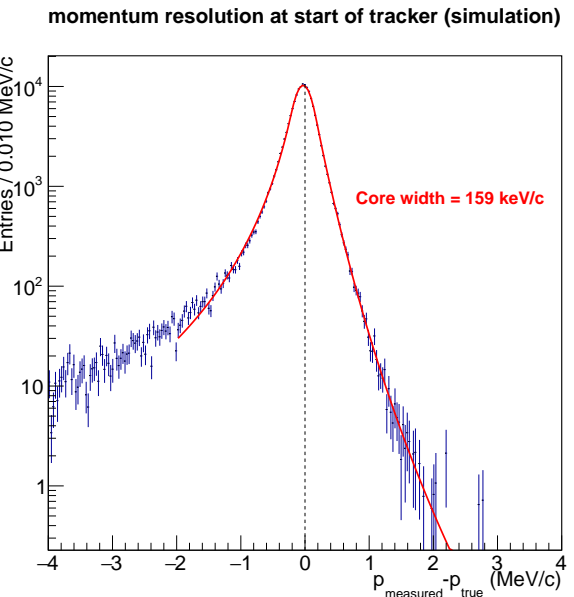

Figure 8. Distribution of the difference between the reconstructed and generated momenta for a CE sample with full Mu2e simulation.

be provided by a Fluorinert liquid circulated in small aluminium tubing in front of the crystals. The Fluorinert will be activated by neutrons from a Deuterium-Tritium generator to produce a $6 \mathrm{MeV} \gamma$ signal. The gain monitoring will be performed with a Laser Flasher system.

A large calorimeter prototype, named Module-0, was assembled in May 2017 at the end of a long selection of pre-production components. This prototype was exposed to an electron beam between 60 and $120 \mathrm{MeV}$ at the BTF facility of LNF. Results on energy and time response and resolution [17] were obtained both for particles impinging normally to the calorimeter surface or at a grazing angle $\left(50^{\circ}\right)$ as for CE's in Mu2e. Simulation has been tuned with data and a detailed data-MC comparison performed (see Fig. 10). The Module-0 performance well satisfied the calorimeter requirements. Module- 0 has also been used to evaluate the operations of running in vacuum and at low temperatures. The same detector is now used to test the behaviour at low temperatures of neutron irradiated components. 


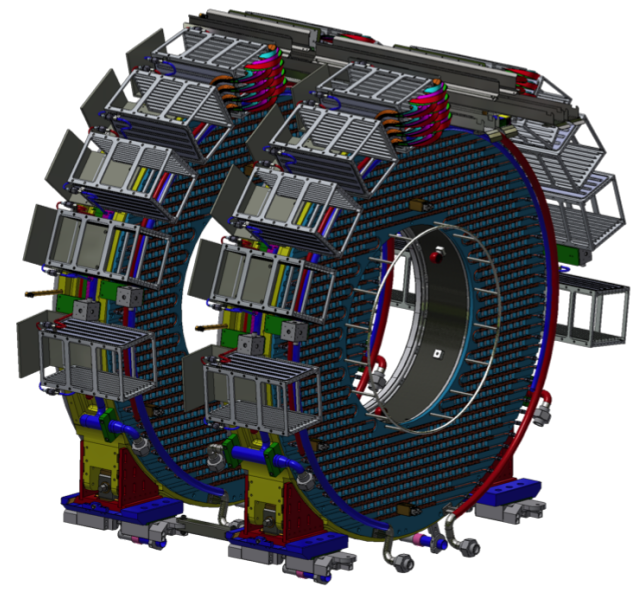

Figure 9. CAD drawing of the calorimeter disks. Calorimeter innermost (outermost) radius is of $351 \mathrm{~mm}(670 \mathrm{~mm})$. Layout of the digitization crates is also shown.

The production of CsI crystals and SiPMs is in good shape. $85 \%$ of crystals have been delivered and tested while the entire SiPMs' production has been completed with very satisfactory results. The prototypes of sensors, FEE and digital electronics (DIRAC) have all been exposed to large ionization doses and neutron fluence in order to select or qualify rad-hard components. The tenders for the main mechanical components, FEE and DIRAC boards are under way at the moment of writing. We expect to start assembling the first calorimeter disk in spring 2020.

One major background source for Mu2e is due to cosmic ray muons producing false $\mathrm{CE}$ candidates when interacting with the detector materials. These events occur at a rate of approximately one/day. In order to reduce their contributions to $\mathrm{O}(0.1)$ event in the entire experiment lifetime, the external area of the DS and a part of the TS (Fig. 11.top) are covered by a Cosmic Ray Veto (CRV) system. The requirement for this system is to obtain a vetoing efficiency of at least $99.99 \%$ for cosmic ray tracks while withstanding an intense radiation environment. Comprised of four staggered layers of extruded plastic scintillation counters (see Fig. 11.bottom), the CRV [18] utilizes two embedded $1.4 \mathrm{~mm}$ diameter Wavelength Shifting Fibers/counter. Each fiber is readout by means of $2 \times 2 \mathrm{~mm}^{2}$ Hamamatsu SIPMs. To achieve the required coverage a total of 5504 counters are needed, organized in 86 modules of six different lengths for a total surface coverage of $327 \mathrm{~m}^{2}$. Test beams on full size prototype have been carried out with satisfactory results demonstrating that the needed light yield can be reached [19]. The average number of photoelectrons observed at $1 \mathrm{~m}$ distance from the readout provides a safety factor of $\sim$ $50 \%$ with respect to the requirement. Irradiation of SiPMs due to neutrons could deteriorate the sensors response and increase the detector occupancy and dead-time so that shielding with concrete blocks (or borated concrete blocks) is mandatory.
The di-counters' assembly started in June 2018 and, at the moment of writing, around half of them have been produced. Production of photosensors, electronics and modules' assembly are also well underway. A test stand with cosmic rays is used to control the modules after production. An example of a cosmic ray event, as recorded by the test stand and by the CRV module under test, is shown in Fig. 12.
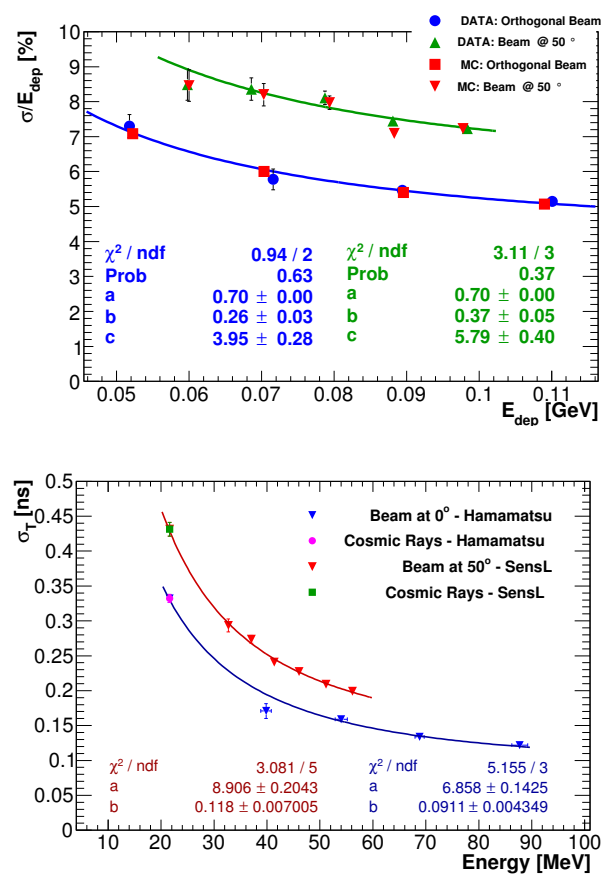

Figure 10. (Top) energy resolution as a function of beam energy for straight electrons (blue points) and electrons impinging at $50^{\circ}$ (green points). Simulation curves are overimposed. (Bottom) timing resolution as a function of beam energy for straight electrons (blue points) and electrons impinging at $50^{\circ}$ (red points).

An extinction monitor detects scattered protons from the production target to evaluate the fraction of out-of-time beam. A High Precision Germanium Detector and a $\mathrm{LaBr}$ crystal are used to monitor the stopping rate in the aluminum target. The latter number is needed to normalize the conversion rate. Further information can be found in Ref. [10] .

\subsection{Reconstruction of CE candidates}

At $100 \mathrm{MeV}$, the momentum resolution is dominated by multiple scattering and fluctuations of the energy loss in the stopping target and in the tracker. By performing a full tracker simulation, a CE tracking reconstruction efficiency of $9 \%$ is obtained for good quality tracks with a minimum of 25 hits/track. The resolution (Fig. 8) is well parametrized by a Crystal Ball function with a negative bremsstrahlung tail, a gaussian core of $159 \mathrm{keV}$ and a long exponential positive tail. The irreducible background for the $\mathrm{CE}$ is provided by DIO electrons that have to be distinguished by the mono-energetic electron signal. The finite tracking resolution and the positive reconstruction tail 

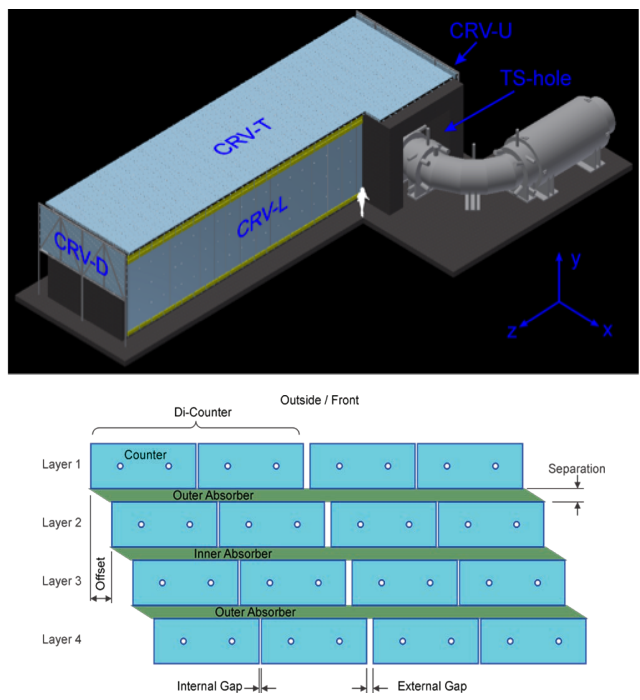

Figure 11. (Top) drawing of the CRV coverage of DS and DS-TS entrance. (Bottom) layout of a CRV module.

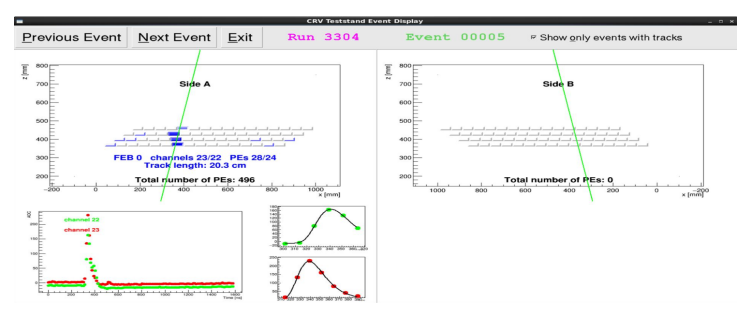

Figure 12. Example of event display at the Cosmic Ray test stand used to qualify CRV modules.

\begin{tabular}{lll}
\hline Source & Bkg process & Yield \\
\hline Intrinsic & Decay in Orbit & $0.14 \pm 0.11$ \\
& Rad. Muon Capture & $0.000 \pm 0.004$ \\
Late Arriving & Rad. Pion capture & $0.025 \pm 0.003$ \\
& Muon decay in flight & $<0.003$ \\
& Pion decay in flight & $0.000 \pm 0.001$ \\
& Beam electrons & $(2.5 \pm 1.2) \times 10^{-4}$ \\
Miscellaneous & Antiprotons & $0.047 \pm 0.024$ \\
& Cosmic rays & $0.24 \pm 0.07$ \\
\hline & Total & $0.46 \pm 0.13$ \\
\hline
\end{tabular}

Table 1. Expected background list as evaluated by the full Mu2e simulation for a three years physics running at full intensity.

have a large effect on the DIO falling spectrum that translates to a residual contamination in the signal region. A full simulation of the experiment has been carried out in the following conditions: (i) $3.6 \times 10^{20}$ protons on target (ii) a corresponding number of $6 \times 10^{17}$ muon stops and (iii) $R_{\mu e}$ of $2 \times 10^{-16}$. A momentum signal window has been selected by optimizing signal over background. The background contribution estimate is presented in Tab. 1, for a total background of 0.46 events. The largest contribution comes from cosmic rays (0.24 events) and DIO's (0.14 events). This counting corresponds to set a limit on
$R_{\mu e}$ below $8 \times 10^{-17}$ at $90 \%$ C.L., in good agreement with the experiment design goal. The number of reconstructed signal events to claim a discovery is 6.66 .

Fig. 13 shows instead the quality of data reconstruction after the Mockup Data Challenge carried out in 2018. On the left plot, the track momentum distribution is shown for a simulation of 5 days Mu2e running without application of any analysis cut. Contribution from DIO's, RPC's, RMC's and cosmic rays is evident. Even in this situation, a CE signal with a BR x10 times better than nowadays limit is visible in the left plot on top of a flat background. The situation improves drastically when the analysis cuts on timing, track, CRV and trigger are added. A clear signal, with practically no surrounding background, stands up in front of an impressive DIO's falling spectrum.

\subsection{Experiment status and schedule}

At the moment of writing the Mu2e experiment has obtained the CD-3 for all systems and its construction is in full swing. The heart of the Mu2e apparatus is provided by the superconducting magnetic system whose design, fabrication, assembly and commissioning drives the experiment schedule. The status of the magnet construction is satisfactory although some delays have been accumulated during the past two years. An international bid for the DS and PS has been concluded and the construction phase for the large magnets is started by General Atomics, San Diego, USA in an assembly facility located in Tupelo (MI). After a long phase of startup, the correct procedure of winding the superconducting cables has been engineered and tested. In Fig. 14.left, a picture of the winding carried out on one of the parts of the DS magnet is shown. For the TS, after the construction of one module prototype by ASG Superconducting, in collaboration with the INFN group of Genova, the contract has been awarded to ASG. All the 52 superconducting coils have been completed and tested in Genova and more than 50\% of the 27 units ( 1 or 2 coils in a mechanical structure) have been delivered to Fermilab. These units are being fully qualified, as shown in Fig. 14.right, with a set of warm/cold/quench tests in a dedicated facility (HAB). The experimental hall has been completed and rapid progresses achieved on the installation of infrastructures (TDAQ room, cables tray, ) with the plan of completing the routing of service cables for summer 2020. The schedule foresees a completion of the detectors by the end of 2020 and the start of their installation in 2021. A long phase of detector commissioning (1-1.5 years) with cosmic rays will follow to complete the functionality of the detector train in the extracted position before inserting it inside the DS. In the meanwhile commissioning of the solenoids and measurement of the magnetic fields will be carried out. The CRV installation will proceed as soon as all the operations on the solenoids are completed. Commissioning with beam is expected by the end of 2022 followed by physics data-taking running soon after. 

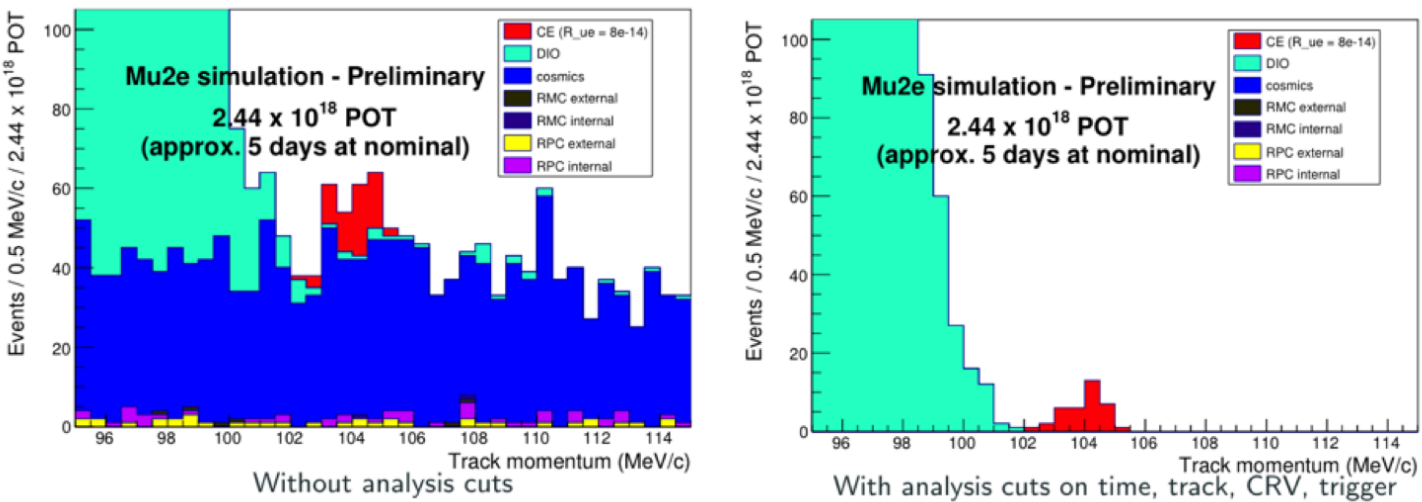

Figure 13. Momentum distribution of reconstructed electrons for a full simulation of 5 days Mu2e running. The signal in red refers to a rate for the conversion of $R_{\mu e}=8 E^{-14}$, i.e. 10 times smaller than current limits. Different backgrounds are represented with different colours as in the captioning: (left) for tracks without analysis cuts, (right) for tracks with analysis cuts on time, track, CRV, trigger. $\mathrm{Mu} 2 \mathrm{e}$ data taking corresponds to around three years of run at full intensity.
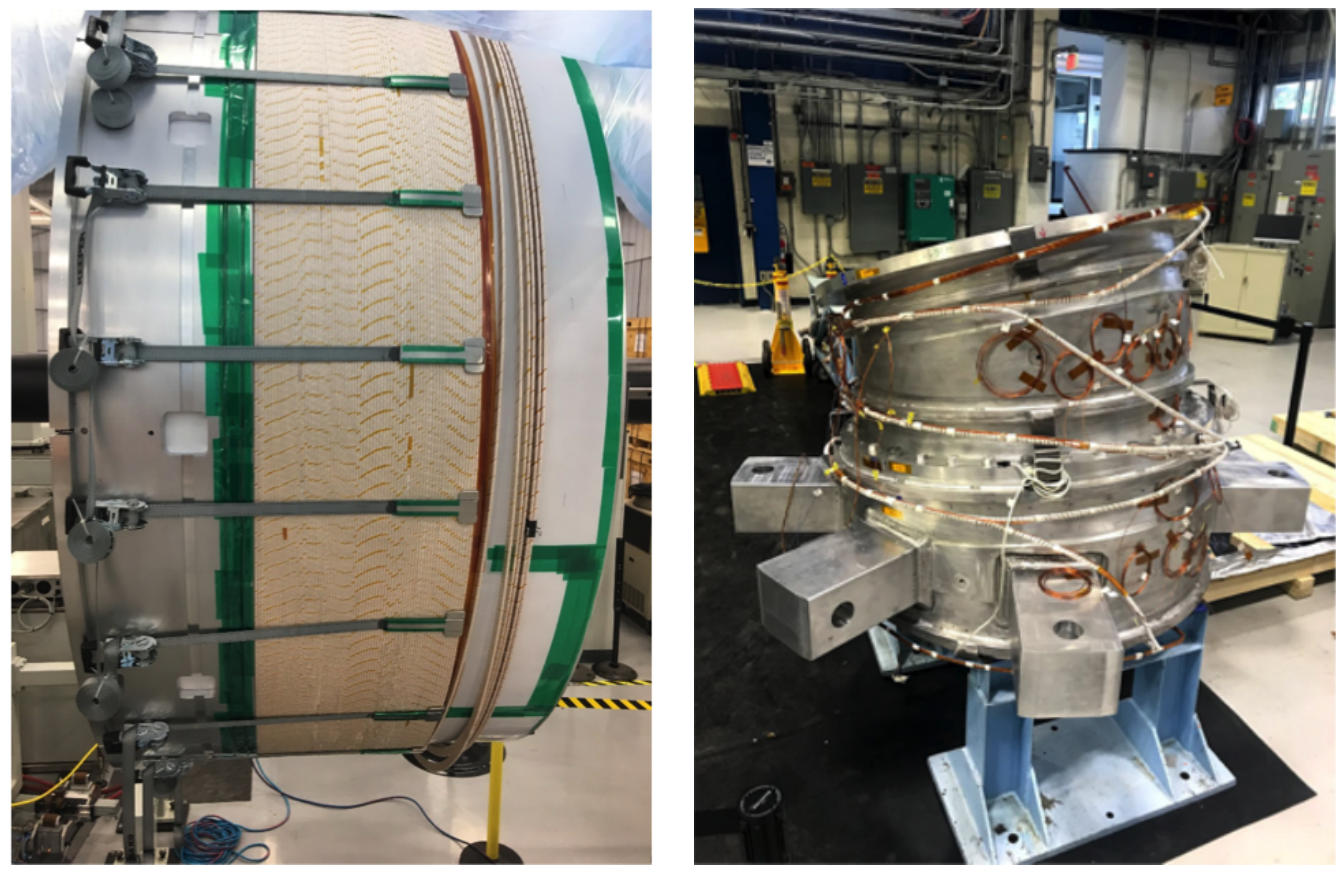

Figure 14. (Left) picture of the coil winding carried out on one of the parts of Detector Solenoid magnet, (right) TS units number 4 during measurements at the HAB facility.

\section{Conclusions and perspectives}

The Mu2e experiment design and construction proceeds well and is scheduled to be commissioned with beam by the end of 2022. It will exploit the world's highest intensity muon beams of the Fermilab Muon Campus to probe cLFV with a single event sensitivity of $3 \times 10^{-17}$ (or set an upper limit on the conversion rate $<8 \times 10^{-17}$ at $90 \%$ C.L.) thus improving the previous sensitivity by four orders of magnitude. A low mass straw tube tracker, a pure CsI crystal calorimeter with SiPM readout and a high efficiency Cosmic Ray Veto have been designed to satisfy the demanding experimental requirements. For the long term future ( $>2028$ ) the possibility of a Mu2e phase- 2 is being explored with the goal of increasing the sensitivity by an additional factor of 10 . This can be obtained with a higher beam intensity and a detector capable of handling the increased accidental activity from muon capture. In this context, an option based on the new Proton Improvement Plan, PIP-2, is being studied.

\section{Acknowledgments}

We are grateful for the vital contributions of the Fermilab staff and the technical staff of the participating institutions. This work was supported by the US Department onf Energy; the Italian Istituto Nazionale di Fisica Nucleare; the US National Science Foundation; the Ministry of Educa- 
tion and Science of the Russian Federation; the Thousand Talents Plan of China; the Helmholtz Association of Germany; and the EU Horizon 2020 Research and Innovation Progam under the Marie Sklodowska-Curie Grant Agreement N.690385. Fermilab is operated by Fermi Research Alliance, LLC under Contract No. De-AC02-07CH11349 with the US Department of Energy.

\section{References}

[1] S.M.Bilenky et al., Phys. Lett. B67, 309 (1977)

[2] W. Marciano et al., Ann. Rev. Nucl. Sci 58 (2008).

[3] L. Cabibbi and G. Signorelli, Riv. Nuovo Cimento 41 71 (2018).

[4] A. Baldini et al., Eur. Phys. J. C 76434 (2016).

[5] U. Bellgardt et al. (The Sindrum Collaboration), Nucl. Phys. B299 1 (1988).

[6] W.Bertl et al. (The Sindrum-II collaboration), Eur. Phys. J C47 337-346 (2006).

[7] Proceedings from the same report.

[8] A. Baldini et al. (The MEG-II collaboration), Eur. Phys. J C 78380 (2018).
[9] A. Blondel et al. (The Mu3e collaboration), arXiv:1301.6113 (2013).

[10] The Mu2e Collaboration, FERMILAB-TM-2594, ArXiv:1501.05241 (2015).

[11] R. Abramishvili et al. (The COMET collaboration), http://comet.kek.jp/Documents_files/PAC-TDR2016/COMET-TDR-2016_v2.pdf.

[12] A. De Gouvea et al., Prog. Part. Nucl. Phys. 71,7592 (2013).

[13] A. Baldini et al. arXiv:1812.06540 (2018).

[14] R.M. Dzhilkibaev and V.M. Lobashev, Sov. J. Nucl. Phys. 49 (2) 384 (1989).

[15] M.J.Lee for the Mu2e Collaboration, Nucl. Part. Phys. Proc., 273-275 2530 (2016).

[16] N. Atanov et al. (Mu2e calorimeter group), IEEE TNS 652073 (2018).

[17] A. Atanov et al. (Mu2e calorimeter group), Nucl. Instr. Meth. A 936, 94 (2019).

[18] A. Artikov et al. FERMILAB-PUB-17-386-PPD, ArXiv:1709.06587 (2015).

[19] G. Blazey et al., Nucl. Inst. Meth. A 927 463-470 (2019). 